\title{
Recommended Conventions for Defining Transition Moments and Intensity Factors in Diatomic Molecular Spectra
}

\author{
Ellis E. Whiting \\ Ames Research Center, NASA, Moffett Field, California 94035 \\ Aert SchadeE \\ "Sonnenborgh" Observatory, The Astronomical Institute at Utrecht, Zonnenburg 2, \\ 3512 NL Utrecht, The Netherlands \\ JEREMY B. TATUM \\ University of Victoria, Victoria, British Columbia, Canada \\ JON T. HOUGEN \\ National Bureau of Standards, Washington, D. C. 20234
}

AND

RalPH W. Nicholls

Center for Research in Experimental Space Science, York University, Downsview, Ontario, Canada

Two recommendations are made that can eliminate persistent confusion in the study of diatomic spectroscopy by providing uniform and consistent definitions of the electronic transition moments and the rotational line intensity factors. First, it is recommended that the equation for the line strength of a single rotational line be adopted to specify the relationship between the electronic transition moment and the rotational line intensity factor. Second, it is recommended that the electronic transition moment operator for perpendicular transjtions be defined by $\left(1 / 2^{1 / 2}\right)\left(\mu_{x} \pm i \mu_{y}\right)$. The adoption of these conventions results in a value of $(2 S+1)(2 J+1)$ for the sum rule of the rotational line intensity factor for $\Sigma^{ \pm} \leftrightarrow \Sigma^{ \pm}$ transitions and a value of $2(2 S+1)(2 J+1)$ for the sum rule for all other spin-allowed transitions.

\section{INTRODUCTION}

Historically, electronic transition moments have been determined by one group of scientists and rotational line intensity factors (also known as Hönl-London factors) by a second group. Thus, the user interested in absolute spectral intensities is required to exercise extreme caution to ensure that compatible values of these parameters are used. In many cases factor-of- 2 errors have been made because of confusion with regard to the sum rules for rotational line intensity factors and the definitions of published electronic transition moments. This bothersome 
situation could well become more acute in the future because electronic transition moments can now be calculated accurately by the methods of quantum chemistry. Obviously, the minimum requirement for eliminating ambiguities that are present in the calculation of absolute intensities is for all authors to define fully the parameters that they publish. However, this will not eliminate the confusion arising from the use of different definitions or different notations for what is essentially the same physical parameter. Thus, we believe there is a need for a few additional standard definitions or conventions in the theory of molecular spectra.

This paper contains two recommendations that we believe would be helpful if universally applied. One is the adoption of a standard expression that specifies the interdependence of the electronic transition moment and the rotational line intensity factor; the other is the adoption of standard definitions of the operators for the electronic transition moments. The combination of these two conventions leads to a simple and consistent sum rule for the rotational line intensity factors for spin-allowed transitions.

Anyone desiring further information about the theoretical details on which these recommendations are based is referred to Refs. $(1,2)$. Anyone desiring to convert the electronic transition moments to other equivalent parameters, such as Einstein $A$ coefficients, band strengths, and oscillator strengths, in a fashion consistent with the definitions made herein, is referred to Ref. (3).

\section{RECOMMENDATIONS}

A single rotational line is usually the simplest spectral element observed and measured in experimental applications, and is the feature we believe provides the most natural starting point to define the electronic transition moments and the rotational line intensity factors. It seems unnecessary to develop standards (for these parameters) based on line components (transitions between degenerate magnetic substates) or on the hyperfine structure; and to develop them based on a group of lines (composite line) requires additional approximations that can obscure the principal issues.

A single rotational line is produced by the allowed transitions between all the magnetic substates of two rotational $J$ levels. Each $J$ level, in an electronic state $n$, is specified by the vibrational quantum number $v$, the resultant angular momentum quantum number without nuclear spin $J$, a single component of the spin multiplet (specified by $\Sigma$ in Hund's case ( $a$ ) coupling, for example), and the lambda substate $p$ due to $\Lambda$-type doubling. That is, a $J$ level is specified fully by $(n, v, J$, $\Sigma, p)$. At the risk of being redundant, we note that a lambda doublet in a spectrum is composed of two rotational lines. Also note that an atomic line is formed by the transitions between two atomic $J$ levels (see Ref. $(4$, p. 97)). This analogous definition of atomic and rotational lines provides a natural conceptual link between atomic and molecular spectra.

The line strength defined by Condon and Shortley (see Ref. (4, p. 98)) for atomic lines can be applied directly to rotational lines:

$$
S_{J^{\prime} J^{m}}=\sum_{M^{\prime} M^{\prime \prime}}\left|\left\langle\psi_{M^{\prime}}|\boldsymbol{\mu}| \psi_{M^{\prime \prime}}\right\rangle\right|^{2},
$$


where $\boldsymbol{\mu}$ is the total transition operator, and $\psi_{M^{\prime}}$ and $\psi_{M^{\prime \prime}}$ are the wavefunctions for magnetic substates of the upper state $\left(J^{\prime}\right.$ level) and of the lower state $\left(J^{\prime \prime}\right.$ level). In this note, we shall restrict $\boldsymbol{\mu}$ to be the electric dipole operator, that is, $\boldsymbol{\mu}$ $=\Sigma e_{i} \mathbf{r}_{i}$, where $\mathbf{r}_{i}$ is a vector from the point at which the dipole moment is being defined to the charge $e_{i}$.

Assuming isotropic excitation and unpolarized radiation, one can write Eq. (1) in the Bron-Oppenheimer approximation for rotational lines as (see Ref. (I))

$$
S_{J^{\prime} J^{\prime \prime},}=q_{v^{\prime} v^{\prime \prime}}\left|R_{\rho}\right|^{2} \mathscr{F}_{J^{\prime} J^{\prime \prime}},
$$

where $q_{v^{\prime} v^{\prime \prime}}$ is the Franck-Condon factor [dimensionless], $R_{e}$ is the electronic transition moment $[\mathrm{C} \mathrm{m}]$, and $\mathscr{S}_{\mathrm{J}^{\prime \prime}}$ is the rotational line intensity factor [dimensionless]. To reduce the chance for misunderstanding, the line strength in Eq. (2) is incorporated in the following equation for the power emitted per unit volume by a rotational line into $4 \pi \mathrm{sr}$, due to spontaneous transitions from an upper $J^{\prime}$ level to a lower $J^{\prime \prime}$ level:

where

$$
E_{J^{\prime} J^{\prime \prime}}=\frac{64 \pi^{4}}{\left(4 \pi \epsilon_{0}\right) 3 c^{3}} \frac{n_{J^{\prime}}}{\left(2 J^{\prime}+1\right)} \nu_{J^{\prime} J^{\prime} q_{v^{\prime} \nu^{\prime \prime}}}\left|R_{e}\right|^{2} \mathscr{S}_{J^{\prime} J^{\prime \prime}},
$$

$$
\begin{aligned}
E_{J^{\prime} J^{\prime \prime}} & =\text { power per unit volume }(\mathrm{W} \mathrm{m}-3), \\
n_{J^{\prime}} & =\text { number density of } J^{\prime} \text { level }\left(\text { particles } \mathrm{m}^{-3}\right), \\
\nu_{J^{\prime} J^{\prime \prime}} & =\text { frequency of emitted radiation }\left(\mathrm{sec}^{-1} \mathrm{or} \mathrm{Hz}^{\mathrm{H}}\right), \\
\epsilon_{0} & =\text { permittivity of a vacuum }\left(8.854 \times 10^{-12} \mathrm{C}^{2} \mathrm{~J}^{-1} \mathrm{~m}^{-1}\right), \\
c & =\text { speed of light }\left(\mathrm{m} \mathrm{sec}^{-1}\right) .
\end{aligned}
$$

Our first recommendation for standardization is that Eq. (2) for the line strength be adopted as the defining expression for the relationship between $R_{e}$ and $\mathscr{S}_{\mathrm{J}^{\prime} \mathrm{j}}$. It is simple and it applies to a single rotational line. We recommend avoiding using an expression for a composite line, such as both rotational lines in a lambda doublet.

Strength expressions for composite lines, or complete vibrational bands, can be derived from equations such as Eq. (3) by summing the intensity, power, etc., that is emitted or absorbed by each of the rotational lines and then making the appropriate approximations for average frequencies, etc. The reverse process, starting from an expression for a composite line strength and working backward to express the line strength for a single rotational line, is much more likely to lead to the kind of ambiguities mentioned in the Introduction.

Equation (2) requires that the electronic transition moment used be compatible with the rotational line intensity factor. These parameters, or the operators that produce them, are defined simultaneously by splitting the total transition operator into separate electronic and rotational parts (see Eqs. (25)-(36) of Ref. (1)). This procedure would be straightforward except that the total transition operator contains a constant factor and there is no inherent "right" way for distributing this constant between the two operators.

In the case of parallel transitions $(\Delta \Omega=0$; or $\Delta \Lambda=0$ for spin-allowed transi- 
TABLE I

Recommended Electronic Transition Moments and Corresponding Sum of Intensity Factors for Spin-Allowed Transitions ${ }^{a}$

\begin{tabular}{|c|c|c|c|}
\hline$\Delta \Lambda$ & $\mathrm{R}_{\mathrm{e}}$ & $X^{\prime} \leftarrow Y^{\prime \prime}$ & $\Sigma \mathscr{S}_{J^{\prime} J^{\prime \prime}}$ \\
\hline \multirow[b]{2}{*}{0} & \multirow[b]{2}{*}{$\left\langle\Lambda^{\prime} \mathrm{S} \Sigma^{\prime}\left|\mu_{z}\right| \Lambda^{\prime \prime} \mathrm{S} \Sigma^{\prime \prime}\right\rangle$} & $\Sigma^{ \pm} \leftrightarrow \Sigma^{ \pm}$ & $(2 \mathrm{~S}+1)(2 \mathrm{~J}+1)$ \\
\hline & & $\begin{array}{l}\Pi \leftrightarrow \leftrightarrow \Pi \\
\Delta \leftrightarrow \Delta\end{array}$ & $2(2 \mathrm{~S}+1)(2 \mathrm{~J}+1)$ \\
\hline \pm 1 & $\left\langle\Lambda^{\prime} S \Sigma^{\prime}\left|\frac{1}{\sqrt{2}}\left(\mu_{\mathrm{x}} \pm i \mu_{\mathrm{y}}\right)\right| \Lambda^{\prime \prime} S \Sigma^{\prime \prime}\right\rangle$ & $\begin{array}{l}\Sigma \leftrightarrow \Pi \Pi \leftrightarrow \leftrightarrow \Sigma \\
\Pi \leftrightarrow \Delta \Delta \leftrightarrow \Pi \\
\Delta \leftrightarrow \Delta \leftrightarrow \Delta\end{array}$ & $2(2 S+1)(2 J+1)$ \\
\hline
\end{tabular}

a The electronic wavefunctions, $|\Lambda S \Sigma\rangle$ shown are the nonrotating-molecule electronic bases wavefunctions, appropriate to Hund's case (a) coupling, discussed in Ref. (2). Specifying the electronic transition moments as matrix elements in terms of these electronic basis functions does not restrict the validity of the theory. Any degree of coupling of the angular momenta intermediate between Hund's cases is incorporated into the rotational line intensity factors, as discussed in Refs. $(1,2)$.

tions), the operator for the electronic transition moment is generally accepted to be $\mu_{z}$ and the constant factor in the total transition operator is 1.0. The remaining factor in the total transition operator is, of course, the rotational operator. Thus, in this case, there is no problem with definitions. This specification of the rotational operator for parallel transitions leads to rotational line intensity factors that satisfy simple sum rules. That is, the sum of all rotational line intensity factors from (or to) the group of $\left(2-\delta_{0, \Lambda}\right)(2 S+1) J$ levels with the same value of $J^{\prime}$ or $J^{\prime \prime}$ is $(2 S+1)$ $\times(2 J+1)$ for $\Sigma^{ \pm} \leftrightarrow \Sigma^{ \pm}$transitions and $2(2 S+1)(2 J+1)$ for all other spin-allowed parallel transitions (see Table I). As usual, the Kronecker delta, $\delta_{0, \Lambda}$, equals 1 if $\Lambda=0$ and equals 0 otherwise.

In the case of perpendicular transitions $(\Delta \Omega= \pm 1$; or $\Delta \Lambda= \pm 1$ for spin-allowed transitions), there is no generally accepted definition of the electronic transition moment operator and, thus, no generally accepted method for distributing the constant factor ( $1 / 2$ in this case) in the total transition operator. In fact, most of the ambiguities referred to in the Introduction can be shown to arise from the way the constant factor is distributed for this case.

Our second recommendation is that the constant factor in the total transition operator for perpendicular transitions be distributed so that the resulting sum rule of the rotational line intensity factors is consistent with the sum rule for parallel transitions. This recommendation requires that the electronic transition moment operators for perpendicular transitions be $\left(1 / 2^{1 / 2}\right)\left(\mu_{x} \pm i \mu_{y}\right)$. When the matrix elements are written as shown in Table $\mathrm{I}$, the plus sign applies to $\Delta \Omega=\Omega^{\prime}-\Omega^{\prime \prime}$ $=+1$ transitions and the minus sign to $\Delta \Omega=-1$ transitions. Using these operators, the sum rule of the rotational line intensity factors for all spin-allowed perpendicular transitions is $2(2 S+1)(2 J+1)$; see Table $\mathrm{I}$.

The consistent use of the recommendations made in this paper leads to a single 
expression for the sum rule for all spin-allowed transitions, ${ }^{1}$ that is,

$$
\Sigma \mathscr{S}_{J^{\prime} J^{\prime \prime}}=\left(2-\delta_{0, \Lambda^{\prime}} \delta_{0 . \Lambda^{\prime \prime}}\right)(2 S+1)(2 J+1),
$$

where again the summation is over all allowed transitions from (or to) the group of $\left(2-\delta_{0, \Lambda}\right)(2 S+1) J$ levels with the same value of $J^{\prime}$ or $J^{\prime \prime}$. The sum rule is symmetrical in $J^{\prime}$ and $J^{\prime \prime}$ and, thus, explicit use of the primes on the right-hand side of Eq. (4) has been omitted.

The factor of 2, which appears in the sum rule for all spin-allowed transitions except $\Sigma^{ \pm} \leftrightarrow \Sigma^{ \pm}$, is present because the summation is over both lambda substates of nonsigma states. Note that in $\Sigma \leftrightarrow \Pi$ and $\Pi \leftrightarrow \Sigma$ transitions, even though only the pi state contains two lambda substates, the factor of 2 is present regardless of which state is the initial state. The result unifies the sum rule for all perpendicular transitions and we hope it will eliminate one of the most frequent causes of confusion in molecular spectra; namely, the intuitive belief that the sum rule for $\Sigma \rightarrow \Pi$ or $\Pi \leftarrow \Sigma$ transitions should be one-half that for $\Sigma \leftarrow \Pi$ or $\Pi \rightarrow \Sigma$ transitions. Because this belief has caused such confusion we present in the Appendix an explanation that may make the concept of equal sum rules for these transitions plausible.

Before concluding this paper, there are two other issues we would like to touch upon. First, there are other arguments, not developed here, for the adoption of the factor $1 / 2^{1 / 2}$ in the definition of the electronic transition moment operators for perpendicular transitions; for example, the desirability from a theoretical point of view of using spherical tensors for all such operators. Second, defining the electronic transition moment as a matrix element in terms of the $|\Lambda S \Sigma\rangle$ basis functions provides a convenient method for reporting theoretical values of these parameters in a manner that is consistent with experimental values. A particular concern about the reporting of theoretical electronic transition moments is discussed in the following paragraph.

The electronic basis wavefunctions used in Table I, and discussed fully in Ref. (2), are characterized by either $\Lambda_{+}=+\left|M_{L}\right|$ or $\Lambda_{-}=-\left|M_{L}\right|$ and, thus, enable the electronic transition moments to be defined in the simple manner shown. Further, they provide a direct association with the electronic wavefunctions normally calculated by ab initio methods using spherical atomic basis functions. Most such calculations are made for $\Lambda_{+}$and $\Sigma=S$, that is, $|\Lambda S \Sigma\rangle=\left|\Lambda_{+} S S\right\rangle$. However, some theoretical calculations on diatomic molecules, particularly those employing Cartesian-Gaussian or Gaussian-lobe functions, do not utilize cylindrical symmetry. The resulting wavefunctions, therefore, may not be characterized by the eigenvalues $\Lambda_{ \pm}$; that is, they may not transform like $x \pm i y$. For example, rather than obtaining $\Pi_{+}$and $\Pi_{-}$as degenerate pi substates, one may obtain instead $\Pi_{x}$ and $\Pi_{y}$ as an equivalent pair of degenerate pi substates that transform like the coordinates $x$ and $y$. Then transition matrix elements such as $\left\langle\Pi_{x}|x| \Sigma\right\rangle$ and $\left\langle\Pi_{y}|y| \Sigma\right\rangle$, where $\Sigma$ is a sigma state transforming like the symmetric representation, are the most convenient to calculate. It is straightforward

\footnotetext{
${ }^{1}$ Equation (4) is not valid for the first few rotational levels in any state where the full spin multiplicity is not developed (i.e., $J<\Lambda+S$ ). In this case, the general sum rule for a spin-allowed or spinforbidden transition given by Eq. (75) in Ref. ( 1 ) can be used, as was done in Ref. (5).
} 
to show, for $\Pi \leftrightarrow \Sigma$ transitions, that the matrix element $\left\langle\Pi_{x}|x| \Sigma\right\rangle$ is equal to $\left\langle\Pi_{+}\left|(x+i y) / 2^{1 / 2}\right| \Sigma\right\rangle$. For other transitions, the relationship between matrix elements can be much more complicated. We recommend strongly that all theoretical electronic transition moment matrix elements be reported as shown in Table I, rather than leaving them in a form that requires additional manipulation or conversion.

Much of the discussion presented has been restricted to spin-allowed transitions. The extension to the more complicated spin-forbidden transitions is described in Ref. (I). Analogous results are obtained; that is, the choice of $\mu_{z}$ for the electronic transition moment operator for parallel transitions and $\left(1 / 2^{1 / 2}\right)\left(\mu_{x} \pm i \mu_{y}\right)$ for perpendicular transitions, results in a simple, general sum rule for the rotational line intensity factors that is consistent with Eq. (4) and is valid for parallel and perpendicular transitions and for spin-allowed and spin-forbidden transitions.

\section{SUMMARY}

We believe that the theory of diatomic molecular spectra can be applied in a less confusing way by the adoption of two standard conventions: first, that the line strength expression for a single rotational line given by

$$
S_{J^{\prime} J^{\prime \prime}}=q_{v^{\prime} v^{\prime \prime}}\left|R_{e}\right|^{2} \mathscr{S}_{J^{\prime} j^{\prime \prime}}
$$

be adopted to specify the interdependence of $R_{e}$ and $\mathscr{S}_{J^{\prime} y^{\prime}}$; second, that $\mu_{z}$ and $\left(1 / 2^{1 / 2}\right)\left(\mu_{x} \pm i \mu_{y}\right)$ be adopted as the electronic transition moment operators for parallel and perpendicular transitions. The adoption of these conventions results in the uniform sum rule

$$
\Sigma \mathscr{S}_{J^{\prime} J^{\prime \prime}}=\left(2-\delta_{0, \Lambda^{\prime}} \delta_{0, \Lambda^{\prime \prime}}\right)(2 S+1)(2 J+1)
$$

for the rotational line intensity factors for spin-allowed transitions.

\section{APPENDIX}

The reason that the sum rules for $\Sigma \leftrightarrow \Pi$ or $\Pi \leftrightarrow \Sigma$ transitions are the same, whether the sigma state is the initial or the final state, can be seen by carrying through the analysis using $\left(1 / 2^{1 / 2}\right)\left(\mu_{x} \pm i \mu_{y}\right)$ as the transition moment operators, and then summing the resulting rotational line intensity factors from (or to) any single $J$ level in either state. In this way, one finds the sum for each single $J$ level in the pi state to be $(2 J+1)$, and for $J$ levels in the sigma state, $2(2 J+1)$. Thus, even though there are twice as many $J$ levels, with the same value of $J$, in the pi state as in the sigma state (because of lambda doubling), the sum rule, which sums the intensity factors over all $J$ levels with the same value of $J^{\prime}$ or $J^{\prime \prime}$, is the same regardless of which state is considered.

The result - that the sum of the intensity factors from (or to) any single $J$ level in the sigma state of $\Sigma \leftrightarrow \Pi$ or $\Pi \leftrightarrow \Sigma$ transitions contains an extra factor of 2 -is consistent with the concept that all transitions between single $J$ levels are equivalent. This is shown easily by considering singlet transitions in terms of the $|\Lambda S \Sigma\rangle$ $=|\Lambda 00\rangle$ basis functions. The extension to higher spin states is conceptually straightforward and the transformation from basis functions to properly sym- 


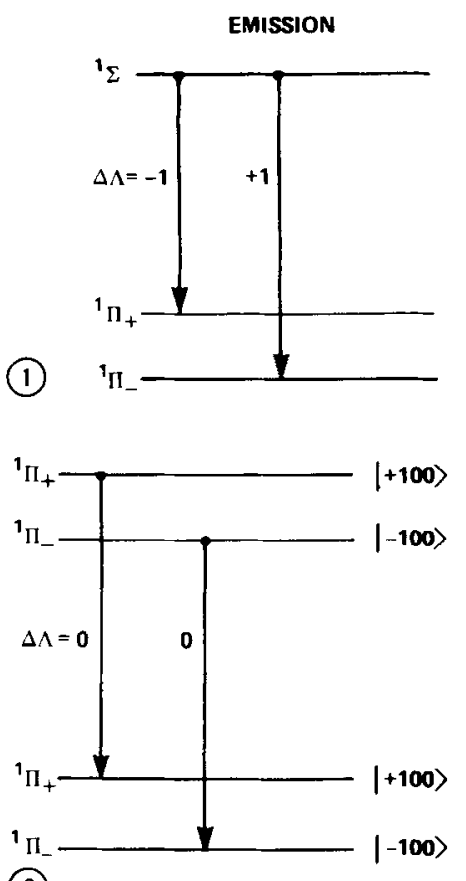

(2)

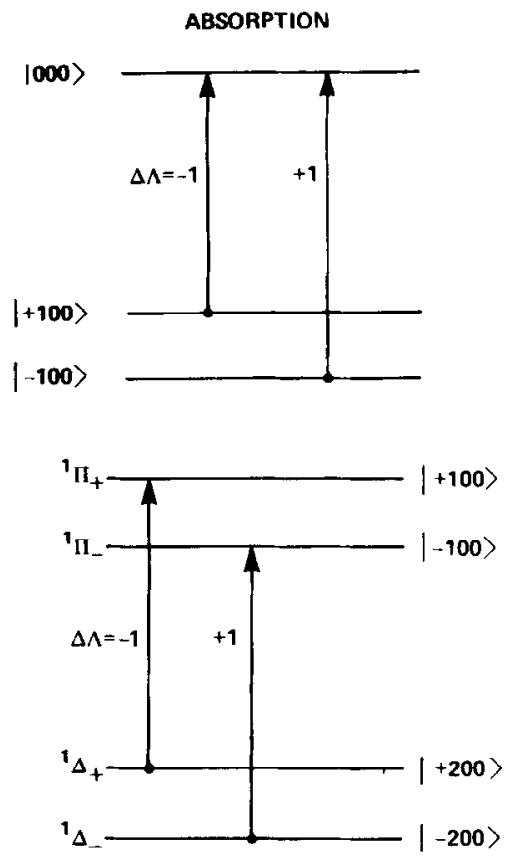

(3)

FIG. 1. Transitions between basis function states: ${ }^{1} \Sigma \leftrightarrow{ }^{1} \prod_{ \pm}$.

FIG. 2. Transitions between basis function states: ${ }^{1} \Pi_{ \pm} \rightarrow{ }^{1} \Pi_{ \pm}$.

FIG. 3. Transitions between basis function states: ${ }^{1} \Pi_{ \pm} \leftarrow{ }^{1} \Delta_{ \pm}$.

metrized wavefunctions is unitary. Unitary transformations do not alter the effect of each transition on the sum rule [see discussion preceding Eq. (56) in Ref. (1)].

In Fig. 1, we show both ${ }^{1} \Sigma \rightarrow{ }^{1} \Pi_{ \pm}$(emission) and ${ }^{1} \Sigma \leftarrow{ }^{1} \Pi_{ \pm}$(absorption) transitions, where as before ${ }^{1} \Pi_{ \pm}$denotes the basis function substates $\left.\mid+100\right)$ and $|-100\rangle$. The corresponding transitions with the ${ }^{1 \Sigma}$ state as the lower state $\left({ }^{1} \Pi_{ \pm} \leftrightarrow{ }^{1} \Sigma\right)$ would be concepiually similar. The figure illustrates that the selection rules $\Delta \Lambda= \pm 1$ permit transitions between the ${ }^{1} \Sigma$ state and both ${ }^{1} \Pi_{ \pm}$substates. Hence, in terms of basis functions, each $J$ level in the ${ }^{1} \Sigma$ state is involved in two transitions, where each $J$ level in the ${ }^{1} \Pi_{ \pm}$substates is involved in only one transition. In all other transitions, ${ }^{1} \Sigma \leftrightarrow{ }^{1} \Sigma,{ }^{1} \Pi \leftrightarrow{ }^{1} \Pi,{ }^{1} \Pi \leftrightarrow{ }^{1} \Delta,{ }^{1} \Delta \leftrightarrow{ }^{1} \Phi$, etc., the selection rules $\Delta \Lambda=0, \pm 1$ permit transitions to occur only from a single basis function substate to a single basis function substate. This is illustrated in Fig. 2 for ${ }^{1} \Pi_{ \pm} \rightarrow{ }^{1} \Pi_{ \pm}$ transitions $(\Delta \Lambda=0)$ and in Fig. 3 for ${ }^{1} \Pi_{ \pm} \leftarrow{ }^{1} \Delta_{ \pm}$transitions $(\Delta \Lambda= \pm 1)$.

Because all transitions are equivalent, an extra factor of 2 must appear in the sum of the intensity factors from (or to) each $J$ level in the sigma state of $\Sigma \leftrightarrow \Pi$ or $\Pi \leftrightarrow \Sigma$ transitions. The recommendations we have made fully account for this effect by including the factor of $1 / 2^{1 / 2}$ in the definition of the transition moment operators for all perpendicular transitions. 


\section{ACKNOWLEDGMENT}

We wish to express our gratitude to our colleague, Stephen R. Langhoff of the Ames Research Center, for the discussion on the symmetry of ab initio wavefunctions.

RECEIVED: March 23, 1979

\section{REFERENCES}

1. E. E. Whiting AND R. W. Nicholls, Astrophys. J. Suppl., No. 235, 27, 1-20 (January 1974).

2. J. T. Hougen, Nat. Bur. Stand. Monog. 115 (June 1970).

3. A. Schadee, J. Quant. Spectrosc. Radiat. Transfer 19, 451-453 (1978).

4. U. Condon and G. H. Shortley, "The Theory of Atomic Spectra," (1967 printing) Cambridge Univ. Press, London, 1935.

5. A. SchadeE, Astron. Astrophys, 41, 203-212 (1975). 